\title{
DEFINICIÓN, DESCRIPCIÓN Y ESTUDIO DE LOS SIMULADORES EN SOFTWARE LIBRE UTILIZADOS PARA EL APRENDIZAJE DE LA FÍSICA
}

\author{
Ariel Adolfo Rodríguez Hernández ${ }^{37}$
}

\begin{abstract}
RESUMEN
Este artículo documenta el estado del desarrollo de simuladores en software libre el aprendizaje de la física y hacer la implementación de un prototipo de repositorio para registrar recursos educativos en Internet, facilitando el acceso a la comunidad .Sus resultados permiten establecer el estado de la simulación en software libre con propósito educativo: por tipo de software, de lenguaje programación, de licencia, de sistema operativo y área de conocimiento, como área piloto se tomó la física. Además, se identifican proyectos de uso de simuladores en USA, Canadá, Argentina, España, Portugal, Francia, Italia, Alemania, Inglaterra, Japón, China, Taiwán, Israel y Colombia, en 50 proyectos y más de 2.097 recursos. Por otro lado, se establece el modelo metodológico para el uso de simuladores en la educación que se sustenta en el aprendizaje activo (cf. Huber, 1997), el aprendizaje basado en problemas (Barrows, 2008), el desarrollo incremental de competencias y el apoyo de la tecnología (TIC's) a fin de hacer uso de este software en la educación. Finalmente, se hizo una prueba piloto de uso de simuladores que permitió identificar: ventajas y desventajas del uso de simuladores. Estos recursos educativos ofrecen alternativas económicas de acceso y excelente calidad para mejorar los procesos educativos.
\end{abstract}

Palabras clave: simulación para educación, uso de simuladores, tecnología educativa.

\begin{abstract}
This paper identity the state of the development of simulations in free software for physics and to development a repository prototype to register educational resources in Internet, facilitating the access to the community.

Their results allow establishing the state of the simulation in software free with educational purpose; for software type, of language programming, of license, of operating system and area of knowledge, as area pilot it was the physics.

It allowed to identify projects of use o simulators in it usA, Canada, Argentina, Spain, Portugal, France, Italy, Germany, England, Japan, China, Taiwan, Israel and Colombia, in 50 projects and already of 2097 resources.
\end{abstract}

37 Universidad Pedagógica y Tecnológica de Colombia. Docente-Investigador, Director Centro Investigación CIDEA/Grupo Investigación TICA. Magíster en Software Libre, Ing. de Sistemas, Email:ariel.rodriguez@uptc. edu.co. Colombia. 
It was define the methodological model for the use of simulators in the education. The methodological model is sustained in the active learning (cf. Huber, 1997), the learning based on problems (Barrows, 2008), the incremental development of abilities and the support of the Information Technology (Іт), in order to make use of this software in the education.

A test pilot of use of simulators was made, this it allowed to identify: advantages and disadvantages of the use of simulators. These educational resources offer alternative of access economic and excellent quality to improve the educational processes.

Key words: simulation for education, use of simulators, educational technology.

Recibido: 28 de abril de 2010

Aceptado: 27 de mayo de 2010

\section{INTRODUCCIÓN}

Este proyecto nace a partir de la necesidad que tienen las Universidades y la sociedad colombiana, en general, frente al tema de laboratorios virtuales de física para estudiantes de Educación a Distancia. El objetivo es implementar, dentro de la educación, el uso de simuladores como apoyo a los procesos educativos.

El tema inicialmente se planteó para todas las áreas pero por lo amplio se decidió tomar la ciencia de la física. Su justificación parte de la necesidad que existe de identificar y conocer el estado actual del desarrollo de simuladores con propósito educativo para la enseñanza de la Física en la educación superior.

El mundo actual viene reconociendo los avances y bondades del software libre, el cual ha incursionado en casi todas las ramas del software, siendo la cuna de estos desarrollos las instituciones educativas, se tiene software libre para procesos administrativos, para desarrollo de soluciones de software, sistemas operativos, administradores de bases de datos, software de ofimática y seguridad. Bajo este panorama, surge la pregunta: en simulación con propósito educativo, ¿el software libre ha tenido impacto?, ¿Qué existe en el mercado? y ¿Cuáles han sido sus resultados?

Esta investigación exploró e identificó el estado de la cuestión y buscó identificar la mayor cantidad de simuladores con propósito educativo para el área de física, con un requisito: que su desarrollo o modelo de distribución sea en software libre. Para ello se tomaron los trabajos de los últimos 10 años. Conciente de la necesidad de no sólo identificar estos recursos, el proyecto incluyó el diseño y desarrollo de un repositorio en línea para ofrecer acceso libre a los recursos. Este repositorio Web se desarrolló en herramientas de software libre y se instaló un administrador/gestor de contenidos que facilitara hacer seguimiento al uso de lo simuladores. 
El proyecto partió de dos preguntas; la primera ¿existe software libre (simuladores) para uso educativo en la enseñanza-aprendizaje de la física? Y la segunda ¿De existir estos recursos cuál es el uso y difusión de los mismos dentro de la comunidad?

Se trabajo durante once meses haciendo búsquedas en Internet, enviando cartas a universidades y comunidades de software libre a fin de obtener fuentes de información sobre el tema. Se inició el diseño y desarrollo del repositorio en línea, el cual se denominó Simula Open source “Open Simula”.

Para organizar la información se definió un sistema de categorización, este incluyó: tipo de lenguaje de desarrollo, tipo de licencia de distribución, área dentro de la física.

\section{METODOLOGÍA}

Para abordar la investigación se definieron, de forma general, las actividades a desarrollar en este proyecto:

- Identificar los sistemas de simulación para laboratorios de física desarrollados en software libre, sus características, funcionalidad, información técnica, código fuente y ejecutables.

- Identificar un modelo metodológico de educación que sustente la utilización de sistemas de simulación para laboratorio de física.

- Definir y diseñar la guía de implementación de un simulador en un curso real.

- Diseñar y desarrollar en software libre el repositorio en línea que contendrá los simuladores, su documentación, fuentes y ejecutables.

- Validar en un curso la utilización de uno de los simuladores y la metodología de educación definida.

- Establecer el diagnóstico e identificar las fortalezas, debilidades y oportunidades de mejoramiento que permitan utilizar de forma más eficiente este tipo de materiales en el aula.

\section{Fases de desarrollo}

\section{Identificación y documentación de recursos de software}

Se buscaron e identificaron las soluciones de software educativo de tipo simulador para laboratorios de física desarrollados en software libre en el ámbito nacional e internacional. Esta etapa o fase incluyó que a cada solución encontrada, y que haya sido desarrollada en los últimos diez años, se le identificaran sus características, funcionalidad, información técnica, el código fuente y los ejecutables en la medida de lo posible, o en su defecto el sitio Web desde el cual se puede utilizar.

Estos programas se seleccionaron previo análisis de las características que deben cumplir en términos del tema que abordan, el cual se desarrolló con base en los contenidos que se 
enseñan en la Física I de los programas de pregrado. Los cuales en particular deben dar tratamiento informático de la simulación de fenómenos relacionados con los temas.

Al mismo tiempo, en esta fase se hizo una revisión de trabajos sobre la problemática didáctica del aprendizaje experimental de la Física, con objeto de identificar los aspectos pedagógicos más importantes que debe cumplir un sistema de simulación para el uso educativo (contenidos teóricos, experiencias virtuales, tareas de desarrollo de destrezas, actividades sobre aprendizaje, metodologías para desarrollo de prácticas apoyadas en simuladores).

\section{Diseño y desarrollo del repositorio en línea}

Previo al diseño y desarrollo se seleccionó como herramienta de desarrollo del repositorio el PHP 5 como lenguaje base de programación para aquellas funciones adicionales que se requieran, y se utilizó para su diseño Web el entorno Mambo o Joomla cuya licencia de uso es General Public Licence (GPL). Como gestor de bases de datos MySQL, como servidor Web Apache. Para configurar el sitio Web se hizo uso de Cpanel.

En esta fase se usó la ingeniería de software para diseñar la solución Web que serviría como repositorio de los simuladores y su respectiva documentación. Diseño que incluyó la integración dentro del sitio Web un sistema gestión de contenidos educativos, que para el proyecto es MOODLE, ya que es software libre, compatible con MySQL, axz $<$ PHP y Apache.

Paralelo al diseño se procedió a desarrollar la solución Web y a iniciar pruebas de los simuladores recopilados, para validar su funcionalidad y el acceso a los recursos que cada elemento del repositorio provea.

\section{Identificación de la metodología educativa y diseño de guías de implementación}

Se requirió que se definiera la metodología educativa a utilizar para validar el uso educativo de simuladores de física en un curso real. Se procedió a definir la población del curso, el nivel, y la modalidad educativa.

Seleccionado como grupo piloto el curso de Física I de la Tecnología en Obras Civiles de la Facultad de Estudios a Distancia de la Universidad Pedagógica y Tecnológica de Colombia, al mismo tiempo se identificó esta metodología educativa de uso, se elaboró la guía de implementación del simulador como material educativo en un curso real, la cual debe ayudar a los alumnos a realizar, de forma práctica, una serie de experiencias de Física, utilizando el simulador seleccionado con el objeto de validar las fortalezas, debilidades y oportunidades de mejoramiento que permitan utilizar más eficientemente este tipo de materiales en la educación superior.

De igual forma, se definieron los indicadores que permiten diagnosticar el uso educativo de los simuladores y el impacto de los mismos en la educación. 


\section{Aplicación}

En esta fases se procedió a incorpora estos recursos a la práctica docente, como herramienta complementaria en la programación de trabajos prácticos de física, en los primeros curso de ingeniería en programas de pregrado.

Y se validó el uso de simuladores. También se buscó analizar la influencia de estas aplicaciones informáticas, y de los materiales didácticos complementarios, en el proceso de aprendizaje que realizan los alumnos a través de las experiencias de laboratorio, evaluando los informes de las sesiones de trabajo realizadas con el software.

\section{Diagnóstico del uso}

Aquí se buscó establecer el diagnóstico frente a la validación del uso de los simuladores en la educación e identificar las fortalezas, debilidades y oportunidades de mejoramiento que permitan utilizar, de forma más eficiente, este tipo de materiales en el aula.

\section{Enfoque metodológico}

En este proyecto se buscó describir la situación actual del tema, identificando los desarrollos de simuladores, haciendo esta investigación de tipo descriptivo, teniendo como propósito primordial establecer el estado de desarrollo del tema: uso educativo de simuladores desarrollados en software libre para física. Tener presente esta consideración es fundamental para comprender los resultados que se dan.

Como uno de los objetivo es crear un banco de información que identifique y presente los desarrollos de software tipo simulador basados en software libre, se dio lugar a establecer las características del análisis de información frente al tema y delimitando el marco general para la investigación.

A continuación presentamos las características que se han definido para la identificación de datos, su tratamiento y análisis. Tipo de software: característica centrada en los tipos de software libre o las condiciones de un software para denominarse software libre. Tomando como concepto que por lo menos cumpliese con una de las libertades ${ }^{38}$ que se han definido en el concepto de software libre. Licencia de distribución del software: se hizo necesario identificar esta característica para, de esta manera, presentar de forma más sólida los resultados. Lenguaje de programación: se buscó establecer cuales lenguajes son los que lideran el desarrollo de simuladores en software libre. Plataforma o Sistema Operativo: se buscó establecer en que sistema operativo puede ser utilizado el software identificado. Área del conocimiento: aunque el proyecto se delimita para sólo física, esta ciencia tiene múltiples áreas para su desarrollo, se identificaron sus áreas fundamentales y se asoció cada resultado a una de estas áreas.

38 Definición de software Libre. Free Software Foundation. http://www.gnu.org/philosophy/free-sw.es.html 
Identificadas las características para hacer el levantamiento de información se hizo necesario categorizar cada una de ellas, definir variables y los indicadores que permitieran valorar los resultados, en la Tabla 1. Variables de investigación se muestran.

Tabla 1. Variables de Investigación

\begin{tabular}{|c|c|}
\hline Característica & Variables \\
\hline \multirow{6}{*}{ Tipo software } & Web o Applet \\
\hline & Aplicación instalable \\
\hline & Sistema Operativo \\
\hline & Directorio \\
\hline & Entorno de desarrollo \\
\hline & LMS Sistema gestor de contenidos \\
\hline \multirow{5}{*}{ Lenguaje programación } & $\mathrm{C}++$ \\
\hline & Java \\
\hline & Python \\
\hline & Perl \\
\hline & Swish / Flash \\
\hline \multirow{4}{*}{ Plataforma } & GNU/Linux \\
\hline & UNIX \\
\hline & Windows \\
\hline & Mac OS \\
\hline \multirow{6}{*}{ Tipo licencia } & GNU GPL \\
\hline & Freeware \\
\hline & LGPL \\
\hline & Creative Commons \\
\hline & Shareware \\
\hline & BSD \\
\hline
\end{tabular}

\section{Universo poblacional y muestra o unidad de análisis}

El universo poblacional está delimitado en términos de tiempo ya que los desarrollos identificados no deben superar los últimos diez años. En volumen de la muestra se buscó primordialmente identificar los desarrollos hechos en instituciones educativas alrededor del planeta. Así como el universo poblacional de las comunidades de software libre y las académicas. La muestra no fue posible establecerla de forma fija, ya que el grado de incertidumbre frente al tema no permitía definir una población, pero sí se definieron las características del objetivo a identificar.

Por otra parte, aunque se buscó identificar proyectos desarrollados en idioma español, se consideró prudente para el análisis objetivo incluir otros idiomas a fin de obtener resultados que lleven a un estudio más completo. 


\section{Elaboración, selección y diseño de instrumentos}

Para identificar proyectos y trabajos relacionados con el objeto del proyecto y documentarlos se diseño una matriz tipo hoja de cálculo incluyendo las características y variables a identificar en el proceso de búsqueda de información,

El instrumento utilizado fue una hoja de registro de resultados, la cual se utilizó en el proceso de identificación y permitió analizar y procesar los datos obtenidos según las necesidades y objetivos definidos por el proyecto.

Esta hoja de registro fue diligenciada para cada proyecto y para cada simulador reportado pro las universidades y organizaciones que colaboraron en la identificación de estos trabajos.

\section{Prueba piloto y validez}

La prueba piloto se desarrolló durante cinco meses y requirió del envió de la ficha a redes educativas y de investigación que permitieran rápidamente identificar posibles fuentes de información. Por otra parte, se realizó una exploración y búsqueda en la Internet para referenciar los proyectos y desarrollos existentes publicados de forma aislada en la Internet.

Fue prioritario para este trabajo lograr que los proyectos estuviesen referenciados y disponibles en la Internet, con el objeto de revisar estos desarrollos y de esta manera garantizar la disponibilidad de los recursos.

\section{Plan de presentación de resultados}

Para la presentación de resultados se consolidó la información, se procedió a tabularla y organizarla según las características y variables definidas. La síntesis de los resultados se presenta en términos de cantidades, porcentajes y representados gráficamente. Los proyectos de alta relevancia se describen brevemente en el documento completo.

\section{RESULTADOS}

Es importante resaltar que se buscó identificar el lenguaje de desarrollo y se encontró que el mayor porcentaje de trabajos están construidos en lenguaje Java, otros lenguajes utilizados son $\mathrm{C}$ o $\mathrm{C}++$, Python.

\section{Identificación de recursos por tipo de software}

Este análisis permitió determinar la cantidad y el porcentaje de simuladores por clase de software identificado con el objetivo de conocer la tendencia del mercado frente a los tipos de software. 
Analizando la Tabla 2, los desarrollos tipo Applet son los más comunes en la simulación en software libre. La causa de este resultado está en la portabilidad de los applets, su facilidad de ejecutarse sobre la Internet, y la robustez para el manejo matemático de los lenguajes utilizados para estas simulaciones que son Java y Flash (Distribuido gratuitamente, el ejecutable más no el código fuente).

Tabla 2. Identificación recursos por tipo de software

\begin{tabular}{|l|r|r|r|r|}
\hline \multicolumn{1}{|c|}{ Tipo Software } & $\begin{array}{c}\text { Cantidad } \\
\text { Proyectos }\end{array}$ & Porcentaje & N. ${ }^{\circ}$ Objetos & Porcentaje \\
\hline Applet (Java /Flash) & 22 & 44,0 & 2069 & 98,7 \\
\hline Sistemas Operativos & 1 & 2,0 & 1 & 0,1 \\
\hline Aplicación Ejec. & 15 & 30,0 & 15 & 0,7 \\
\hline IDE & 5 & 10,0 & 5 & 0,2 \\
\hline Directorio & 2 & 4,0 & 2 & 0,1 \\
\hline LMS & 5 & 10,0 & 5 & 0,2 \\
\hline Total proyectos & $\mathbf{5 0}$ & & $\mathbf{2 0 9 7}$ & \\
\hline
\end{tabular}

La gráfica 1 muestra cómo la población de simuladores de física desarrollados en software libre se distribuyen según el tipo de software.

Gráfica 1. Porcentaje Identificación recursos por tipo de software

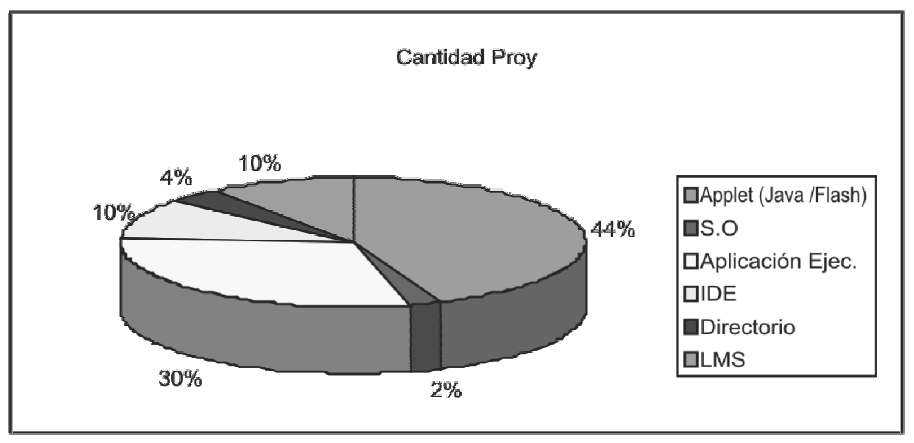

Los simuladores identificados tipo applet desarrollados en lenguaje Java, permiten descargar el código fuente o binario y los archivos de ejecución (.jar). En materia de aplicaciones tipo ejecutable, se pudo establecer que corresponden en su gran mayoría a aplicaciones para simular gráficas de alta complejidad matemática.

Finalmente los entornos de desarrollo tipo IDE para creación de simulaciones en software libre son escasos pero poco a poco se consolidan como el futuro de lo simuladores en de este estilo. En un numeral más adelante se describen con detalle estas soluciones. 
A nivel de directorio Web o repositorios de recursos fue posible identificar dos repositorios de tipo base de datos informativa.

Y a nivel de sistemas operativos de código abierto se identificó el sistema operativo Ing-X, que en su distribución integra los más robustos y complejos desarrollos para simulación. Este proyecto tiene sus orígenes en la Republica de la Argentina e integra más de 15 soluciones de simulación para múltiples áreas del conocimiento científico.

\section{Identificación de recursos según el lenguaje de desarrollo}

Dentro del proceso de investigación y documentación de los proyectos, a cada proyecto se le identificó el lenguaje de programación en el que fue desarrollado. El objeto de este análisis es establecer la tendencia en lenguajes de programación para el desarrollo de simuladores e identificar las causas de su uso. De forma concluyente arroja que el lenguaje Java es el más utilizado para el desarrollo de simuladores, seguido de Flash, C++ y Python. Este último lenguaje está siendo probado en el sistema SimPy = Simulation in Python, proyecto que ha creado una solución para desarrollar simuladores, véase gráfica 2. Porcentajes de simuladores según lenguaje de programación utilizando. Ver Tabla 3.

Tabla 3. Clasificación simuladores según lenguaje de programación

\begin{tabular}{|l|r|r|r|r|}
\hline $\begin{array}{c}\text { Lenguaje } \\
\text { Programación }\end{array}$ & $\begin{array}{c}\text { Cantidad } \\
\text { Proyectos }\end{array}$ & Porcentaje & N. ${ }^{\circ}$ Objetos & Porcentaje \\
\hline Java & 29 & 58,0 & 1880 & 89,7 \\
\hline Python & 6 & 12,0 & 7 & 0,3 \\
\hline Flash & 3 & 6,0 & 198 & 9,4 \\
\hline C++ & 10 & 20,0 & 10 & 0,5 \\
\hline NA & 2 & 4,0 & 2 & 0,1 \\
\hline Total & $\mathbf{5 0}$ & & $\mathbf{2 0 9 7}$ & \\
\hline
\end{tabular}

Java es el lenguaje más utilizado debido a que es multiplataforma. Esto lo hace portable y escalable. Su facilidad para el manejo matemático y gráfico en 2D y 3D integran las complejas operaciones y gráficas que la física requieren para simular eventos. Por otra parte, al poder ejecutarse sobre un navegador de Internet, da lugar a que su distribución y uso sea fácil.

Caso especial es el tema de simuladores desarrollados en Flash, uno de los sistemas de desarrollo de mejor procesamiento gráfico y versátil a la hora de integrarse con la Internet.

La diferencia central de los desarrollos en Java, C++ y Python frente a Flash está en que los 3 primeros lenguajes ofrecen las cuatro libertades establecidas para el software libre, mientras que Flash sólo concede las libertades 1 y 3, ejecución y distribución. Ver gráfica 2. 
Grafica 2. Porcentajes de simuladores según lenguaje de programación utilizando

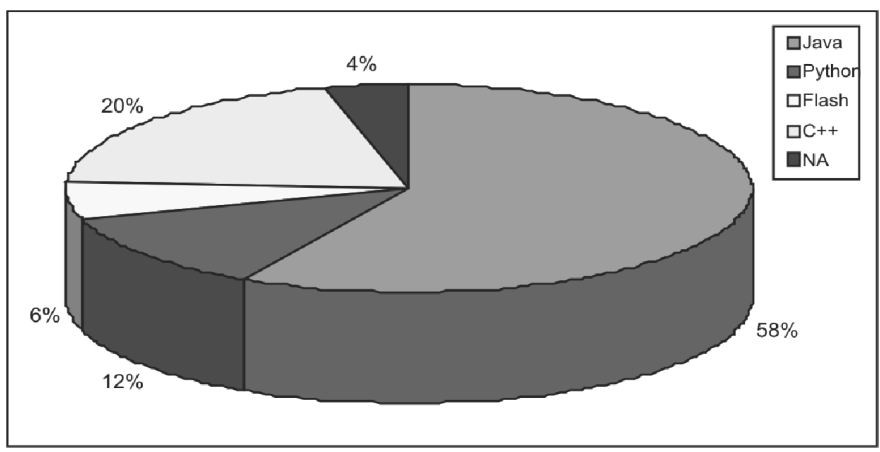

\section{Identificación de recursos según el tipo de licencia de distribución}

Este ítem permitió identificar la tendencia en materia de licenciamiento de los recursos tipo simulador identificados y el nivel de implementación de los compromisos del software libre, según la licencia de distribución de cada recurso, así como las libertades permitidas.

La tabla 4 refleja los resultados de este estudio.

Tabla 4. Clasificación simuladores según tipo Licencia

\begin{tabular}{|l|r|r|r|r|}
\hline \multicolumn{1}{|c|}{ Tipo Licencia } & \multicolumn{1}{c|}{$\begin{array}{c}\text { Cantidad } \\
\text { Proyectos }\end{array}$} & Porcentaje & \multicolumn{1}{c|}{ N. $^{\circ}$ Objetos } & \multicolumn{1}{c|}{ Porcentaje } \\
\hline GPL & 16 & 32,7 & 120 & 5,7 \\
\hline LGPL & 1 & 2,0 & 1 & 0,0 \\
\hline BSD & 2 & 4,1 & 2 & 0,1 \\
\hline Freeware & 28 & 57,1 & 1867 & 89,0 \\
\hline Creative Commons & 2 & 4,1 & 107 & 5,1 \\
\hline Total & $\mathbf{5 0}$ & & $\mathbf{2 0 9 7}$ & \\
\hline
\end{tabular}

Se observa con preocupación que las libertades totales del software libre deben ser abordadas y concertadas a fin de aprovechar las fortalezas de las comunidades de desarrollo en el mejoramiento de estos recursos.

El freeware con un $57.1 \%$ es la forma más común de publicar y poner a disposición del público un software, y la desventaja del mismo es que no cumplen con las cuatro libertades establecidas por el software libre, se ve de igual forma que la licencia GPL ocupa el segundo lugar con un $32.7 \%$, véase la grafica 3 . 
Gráfica 3. Porcentajes de simuladores según tipo licencia con la que se distribuye

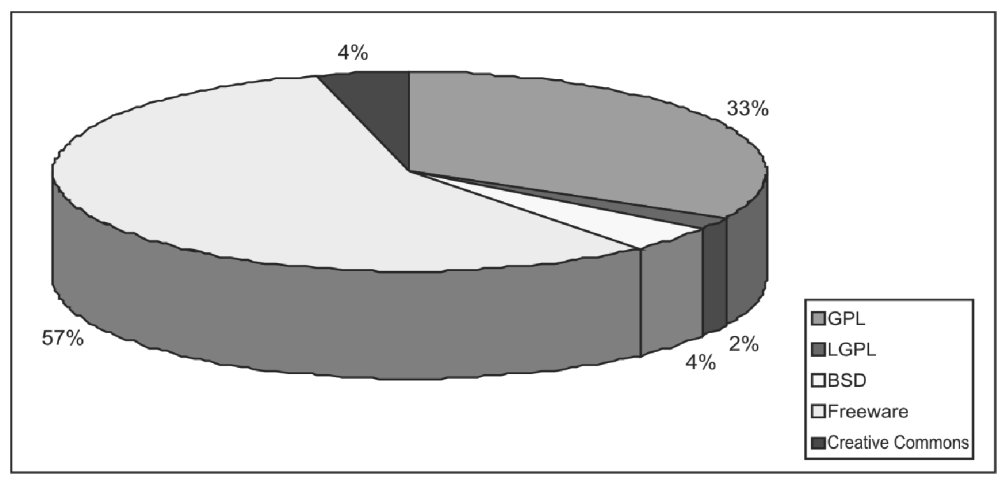

Esto lleva a que se plantee la necesidad de producir bajo un mismo criterio la forma de convertir el freeware en licencia GPL para garantizar las 4 libertades del software libre y potencilizar las ventajas que este modelo de desarrollo ofrece.

\section{Identificación de recursos según plataforma de uso}

Es fundamental identificar el sistema operativo en el que funciona cada simulador, ya que esto asegura la transportabilidad del mismo.

Los resultados descritos en la tabla 5 en forma concluyente identifican que los desarrollos son en un $70 \%$ multiplataforma asegurando con ello el uso de los mismos tanto en GNU/ Linux, como en Windows o incluso Mac OS. Ver n. ${ }^{\circ} 4$. Porcentajes de simuladores según su disponibilidad por Sistema Operativo.

Tabla 5. Clasificación simuladores según los Sistemas operativos para su uso

\begin{tabular}{|l|r|r|r|r|}
\hline \multicolumn{1}{|c|}{ Plataforma } & \multicolumn{1}{c|}{$\begin{array}{c}\text { Cantidad } \\
\text { Proyectos }\end{array}$} & Porcentaje & N. ${ }^{\circ}$ Objetos & Porcentaje \\
\hline GNU/Linux & 8 & 16,0 & 8 & 0,4 \\
\hline Windows & 5 & 10,0 & 202 & 9,6 \\
\hline Multiplataforma & 35 & 70,0 & 1885 & 89,9 \\
\hline Mac OS & 2 & 4,0 & 2 & 0,1 \\
\hline Total & 50 & & 2097 & \\
\hline
\end{tabular}

De manera clara se puede ver que los simuladores desarrollados son multiplataforma: ver gráfica 4. 
Gráfica 4. Porcentajes de simuladores según su disponibilidad por Sistema Operativo

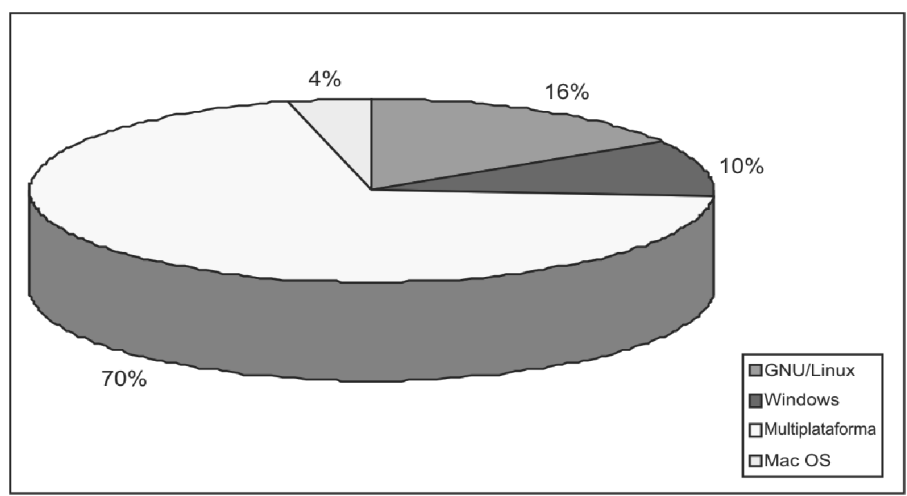

\section{Denominación, ubicación y estructura del repositorio}

El repositorio en línea tiene la siguiente descripción:

Título: Simulación Open Source.

Subtítulo: Repositorio de simuladores basado en software libre.

Dirección URL: http://www.ticagi.org Esta es la dirección general.

Servicios perfil usuario: para acceder al repositorio a modo de consulta de recursos no se requiere autenticación y puede hacer uso de los siguientes servicios:

- Búsqueda por categoría o nombre del recurso.

- Canales RSS de noticias relacionadas con el tema de simulación y otros.

- Sección de enlaces destacados sobre simulación

- Sección para registro como usuario del repositorio y para subir y colaborar en la identificación y referenciando de los recursos tipo simulador.

Este último servicio requiere registro y creación de un usuario para autenticarse y entrar como colaborador en la referenciación de simuladores en software libre.

\section{Diseño del prototipo de guía para uso educativo de simuladores de física}

Identificados los diferentes simuladores desarrollados en software libre, se hace necesario diseñar las respectivas guías para hacer uso educativo de estos sistemas dentro de un curso de física a nivel de educación superior. Frente a este tema son varios los trabajos que se han desarrollado y que se evaluaron para construir la guía y el modelo metodológico para usar simuladores. 
Los trabajos sobre simulación computacional en la enseñanza de la Física (Kofman, H. 2000) y posteriormente los resultados mostrados de su proyecto (Kofman, H. 2003), describen con claridad el alto impacto del simulador como herramienta de apoyo a la educación.

De otra parte, trabajos sobre uso de simuladores en física en Argentina dan cuenta del impacto en la retención y la facilidad que estos recursos brindan para comprender los diferentes eventos que la física estudia (Giuliano, M, 2006).

Los trabajos desarrollados por Rosado, L. y Herreros J.R. (2005), analizan los simuladores, desde la didáctica de la enseñanza de la física y describen las ventajas y desventajas que este tipo de recursos ofrece a la educación; resaltando que para ambientes educativos a distancia o virtuales son una de las mejores opciones para práctica y desarrollo de laboratorios de física.

Por último, los trabajos de Sierra, J. y Perales, F. (2007), permiten evidenciar que los aprendizajes en poblaciones educativas que utilizan el simulador dentro del proceso formativo reflejan mayor entendimiento de las situaciones, tanto desde el punto de vista físico como matemático.

Por otro lado, se hizo necesario reconocer cómo las TIC's pueden apoyar la educación, Franco, I. y Álvarez, F. 2008), el uso de simuladores dentro de ambientes virtuales educativos (Franco, I. y Álvarez, F. 2008) plantean la compatibilidad de estas soluciones como herramientas didácticas de fácil uso y que sin duda ofrecen múltiples mejoras en el proceso de enseñanza a través de la práctica haciendo uso de TIC's.

El proyecto requería definir un prototipo de guía para el uso educativo de simuladores y el trabajo más completo que permitió orientar esta parte del proyecto remitió al autor al proyecto denominado Aprendizaje de la física con modelamiento matemático (Teodoro, V.D. 2003), el cual define un software desarrollado para construir simulaciones y la metodología de implementación de las simulaciones dentro de la educación.

Este tesis posdoctoral en su capítulo 6 "uso de Modellus" (Teodoro, V.D. 2003), describe los referentes teóricos para hacer uso educativo de simuladores y define la estructura general de las guías requeridas para hacer uso de esta solución de software de simulación, razón por la cual se tomó como referencia principal para esta parte del proyecto.

De manera breve una guía metodológica para uso de simuladores requiere:

- Descripción de los objetivos del taller

- Descripción del simulador a utilizar

- Desarrollo conceptual del tema

- Descripción de la metodología de trabajo y los ejercicios a desarrollar

- Descripción del informe a presentar 
Dentro del estudio desarrollado este proyecto es el más completo y documentado en materia de uso educativo de simuladores de física. Se utiliza por tal razón la guía que allí se propone para uso educativo de simuladores, la cual es abierta para ser utilizada con cualquier otro simulador y es el modelo de guía que ha sido utilizado en el desarrollo de prácticas dentro de los cursos de Física I de la Facultad de Estudios a Distancia, con resultados satisfactorios.

Este prototipo debe estar en constante revisión, evaluación y actualización y dependiendo el simulador que se utilice debe ser ajustado en sus componentes según se considere pertinente.

\section{Fortalezas, debilidades y oportunidades de mejoramiento del aprendizaje utilizando simuladores}

Para la validación del uso educativo de los simuladores seleccionados, se tomó el curso de Física I, grupo 550, con 26 estudiantes y grupo 551 con 29. Ambos grupos pertenecen al programa de Tecnología en Obras Civiles en modalidad a distancia.

Para dar inicio a estos cursos, a los alumnos se les brindó una capacitación en la que se familiarizaron con los simuladores, tanto en su proceso de instalación como de uso. Guiados por el docente fueron conociendo el simulador y la forma de interactuar con el mismo.

El grupo experimental estuvo integrado por dos grupos de estudiantes, el primer grupo (550) utilizó el entorno simulación Modellus y el segundo grupo (551) utilizó los simuladores del proyecto physlets (Esquembre, 2004).

Cada entorno es muy diferente ya que el Modellus (Teodoro, V.D. 2003), es un sistema integrado de diseño y desarrollo de simulaciones, en el cual el usuario puede cambiar no sólo las variables sino las condiciones de simulación, incluso de un ejemplo pueden estar creando una nueva simulación. Los physlets son applets con simulaciones prediseñadas en las que el estudiante sólo cambia variables y luego hace la prueba de la simulación con esas variables.

\section{Ventajas y fortalezas}

- El uso de simuladores pone al alcance de cualquier institución educativa las prácticas de física, sin requerir laboratorios complejos y altamente costosos y ofrece al estudiante tener en su propio lugar de residencia el entorno simulado de práctica.

- Los simuladores permiten reproducir fenómenos naturales difícilmente observables de manera directa en la realidad, por motivos diversos: riesgos, costos, escala de tiempo, escala espacial.

- El estudiante prueba sus ideas previas y conocimientos acerca del fenómeno simulado mediante la emisión de hipótesis propias, lo cual redunda en una mayor autonomía del proceso de aprendizaje y el desarrollo del aprendizaje constructivista y significativo. 
- El estudiante puede modificar los distintos parámetros y condiciones iniciales que aparecen en el modelo físico que el simulador ofrece, lo que ayuda a formular sus propias conclusiones a partir de distintas situaciones.

- La simulación ofrece al estudiante amplia variedad de datos relevantes, que facilitan la verificación cualitativa y cuantitativa de las leyes y postulados científicos de las diferentes áreas de la ciencia.

- Los simuladores visualizan gráficas en tiempo real de distintas magnitudes, brindándole al estudiante una noción mucho más real de los fenómenos simulados.

\section{Desventajas y desafíos}

- En general desventajas como tal no se han identificado, realmente la problemática que genera el uso de simuladores está dado hacia algunos desafíos:

Cuando los estudiantes se inician en el uso simuladores suelen, en ocasiones, modificar variables del fenómeno simulado que no son relevantes para contrastar sus hipótesis y que si generan errores en la ejecución del programa, se requiere para prevenir esto ofrecer una adecuado capacitación en el uso de los simuladores y guías claramente definidas.

- Los sistemas de simulación más eficaces desde el punto de vista didáctico son los que implementan una diversidad suficiente de modelos físicos, con distinto nivel de complejidad, como sucede en el entorno de simulación Modellus, en el cual cada modelo físico implementado se asocia con una determinada interfaz informativa para el estudiante, de manera que la secuencia de tareas propuestas requiere que el estudiante siga las indicaciones planteadas para iniciarse en el uso del simulador.

\section{CONCLUSIONES Y FUTUROS TRABAJOS}

\section{Conclusiones}

En el trabajo se propuso identificar y documentar los proyectos de software educativo desarrollados en software libre mediante el desarrollo de un repositorio en línea, igualmente desarrollado en software libre.

Es de precisar que los resultados fruto de esta etapa del proyecto son satisfactorios en cantidad, se identificaron alrededor de 2000 soluciones de software, cada uno documentado y organizado según el área de aplicación.

De este estudio se concluye que los simuladores identificados que cumplan con los criterios de software libre son apenas un $42.4 \%$ del total identificado, situación que lleva a concluir que en 1 actualidad el concepto de software libre bajo los principios que a este lo rigen 
aun no son claros para las comunidades de desarrollo y mucho menos para las instituciones educativas, se requiere mayor difusión de las ventajas que el software libre ofrece.

Se requiere mayor esfuerzo por parte de las asociaciones y fundaciones gestoras y promotoras del software libre y los expertos en el tema para dar a conocer y promover las ventajas del software libre.

Por otra parte, en su gran mayoría, estos simuladores son iniciativas personales e individuales de profesores de diferentes instituciones que movidos por el deseo de mejorar la educación han desarrollado este tipo de recursos y que por la necesidad de los mismos han logrado vincular a estas iniciativas a otros colegas.

Se requiere liderar de forma más organizada y con criterios de calidad de software educativo y de la dinámica de sistemas este tipo de proyectos para que dentro de una comunidad de desarrolladores se consolide un modelo de desarrollo de software educativo libre.

Desde el punto de vista educativo se identificaron varios proyectos que metodológica y pedagógicamente son muy sólidos y pueden ser los derroteros para consolidar el uso educativo de simuladores dentro de la sociedad.

\section{Futuros trabajos}

Identificados los más de 2000 simuladores un trabajo futuro muy importante estaría dado por la validación en términos educativos de estos recursos y bajo los criterios de calidad del software educativo. Este tipo de investigación les permitiría a las organizaciones educativas y docentes tener mejores elementos a la hora de hacer uso de estos recursos.

De otra parte, con la existencia de entornos integrados de desarrollo de software libre se debe realizar el análisis y estudio que lleve a definir la metodología para el desarrollo de simuladores en software libre utilizando estos sistemas y con sus respectivos procesos de validación desde lo pedagógico, el diseño instruccional y las teorías del aprendizaje que sustentan la calidad de estos sistemas.

Se tiene proyectado investigar este mismo tema ampliándolo a otras áreas del conocimiento (química, matemáticas y biología), ya que al desarrollar este proyecto fue posible vislumbrar que hay simuladores para muchas otras áreas, pero que de igual forma podrían identificarse y mediante el repositorio colocarse al alcance de la comunidad.

Un proyecto posterior a este debe tomar estos simuladores y validarlos desde criterios que midan el aprendizaje y su impacto en los procesos de desarrollo de conocimiento. 


\section{REFERENCIAS BIBLIOGRÁFICAS}

ESQUEMBRE, F. (2004). Creación de simulaciones interactivas en Java: aplicación a la enseñanza de la Física. Madrid: Pearson Educación.

FRANCO, I., ÁlVAREZ, F. (2008). Los Simuladores, estrategia formativa en ambientes virtuales de aprendizaje. Revista Virtual Universidad Católica, Vol. 21. Consultado el 10 de octubre de 2009 en: http://www.ucn.edu.co/portal/uzine/volumen21/articulos/3 InvestigaciC3B3n_simuladores.pdf

GIULIANO, M., MARCHISIO, S. Y OTROS. (2006). Diseño de simuladores para un curso a distancia dirigido a docentes de Física de distintas jurisdicciones de Argentina. Current Developments in Technology-Assisted Education. Consultado el 26 de octubre de 2009 en: http://www.formatex.org/micte2006/pdf/2047-2051.pdf

KOFMAN, H. (2003). Integración de las funciones constructivas y comunicativas de las NTICs en la enseñanza de la Física Universitaria y la capacitación docente. Premio del II Concurso "Educación en la red". Consultado el 03 de octubre de 2009 en: http://www. educared.org.ar/concurso-2/resenia/pdf/04-kofman.pdf

KOFMAN, H. (2000). Modelos y simulaciones computacionales en la enseñanza de la Física. Revista Educación en Física Vol.6, 13- 22.

ROSADO, L., HERREROS J.R. (2005). Nuevas aportaciones didácticas de los laboratorios virtuales y remotos en la enseñanza de la física. Consultado el 17 de octubre de 2009 en: http://209.85.165.104/search?qcache:JCAD80KOvJYJ:www.formatex.org/micte2005/286.p df22NuevasaportacionesdidC3A1cticasdeloslaboratorios+virtualesyremotosenlaenseC3B1a nzadelafC3ADsica,22\&hles\&ctclnk\&cd1\&glco\&lrlang_es

SIERRA, J., PERALES, F. (2007). Aprendiendo física en bachillerato con simuladores informáticos. Revista Alambique: Didáctica de las ciencias experimentales, Vol.51, 89-97.

TEODORO, V.D. (2003). Modellus: Learning Physics with Mathematical Modelling. Tesis postdoctoral no publicada. Universidade Nova de Lisboa, Lisboa. Consultado el 19 de septiembre de 2009 en: http://modellus.fct.unl.pt/mod/resource/view.php?id=334 